\title{
Endometriosis-related pneumothorax after in vitro fertilization embryo transfer procedure: A case report
}

\author{
Alessandro Baisi, MD, ${ }^{\mathrm{a}}$ Federico Raveglia, MD, ${ }^{\mathrm{a}}$ Matilde De Simone, MD, PhD, ${ }^{\mathrm{b}}$ \\ Angelo Maria Calati, MD, ${ }^{\mathrm{a}}$ Andrea Leporati, MD, ${ }^{\mathrm{a}}$ and Ugo Cioffi, MD, $\mathrm{PhD},{ }^{\mathrm{b}}$ Milano, Italy
}

Catamenial pneumothorax is a unique clinical entity occurring in ovulating women at the onset of menses. It was considered rare, but in a recent report its incidence amounts to $25 \%$ of spontaneous pneumothorax surgically treated in women. ${ }^{1}$ This justifies an increasing interest on the topic. Despite the fact that catamenial pneumothorax has been known for 5 decades, its cause has not been defined definitively. Thoracic endometriosis is frequently indicated as an implicated factor; however, the pathogenesis and mechanism that lead to thoracic endometriosis and from endometriosis to pneumothorax are still unclear. ${ }^{2,3}$

To our knowledge, we report the first case of a catamenial pneumothorax in a woman who underwent controlled ovarian hyperstimulation for in vitro fertilization embryo transfer (IVF-ET) cycles, and we add some information to clarify its pathogenesis.

\section{CLINICAL SUMMARY}

In October 2006, a 32-year-old woman presented with dyspnea and acute right-sided chest pain. Her clinical history was negative except for a mild pain located on the right side of the back close to the scapula, associated with a cough recurring every menses for 5 years. She underwent an unsuccessful IVF-ET cycle 2 weeks before. In August 2006, she started the hormone therapy and was initially placed on a gonadotropin-releasing hormone agonist to suppress the pituitary activity. Then she received follitropin alpha starting from a fixed daily dose of 150 IU to obtain controlled ovarian hyperstimulation. Finally, chorionic gonadotropin at a dose of $10,000 \mathrm{IU}$ was administered to induce the ovulation. Thirteen oocytes were obtained and 2 fertilized, and 2 were implanted according to Joint Society of Obstetricians and Gynecologists of Canada-Canadian Society of Fertility and Andrology Guidelines. ${ }^{4}$

\footnotetext{
From the Thoracic Surgery Unit, Ospedale San Paolo, ${ }^{a}$ and Department of Surgery, Fondazione IRCCS Ospedale Maggiore Policlinico, ${ }^{\mathrm{b}}$ University of Milan, Milano, Italy.

Disclosures: None.

Received for publication Nov 24, 2008; accepted for publication Dec 19, 2008; available ahead of print June 24, 2009.

Address for reprints: Alessandro Baisi, MD, Thoracic Surgery Unit, Ospedale San Paolo, Via A. di Rudinì, 8, 20142 Milano, Italy (E-mail: alessandro.baisi@unimi.it). J Thorac Cardiovasc Surg 2010;139:e88-9

$0022-5223 / \$ 36.00$

Copyright (c) 2010 by The American Association for Thoracic Surgery doi:10.1016/j.jtcvs.2008.12.056
}

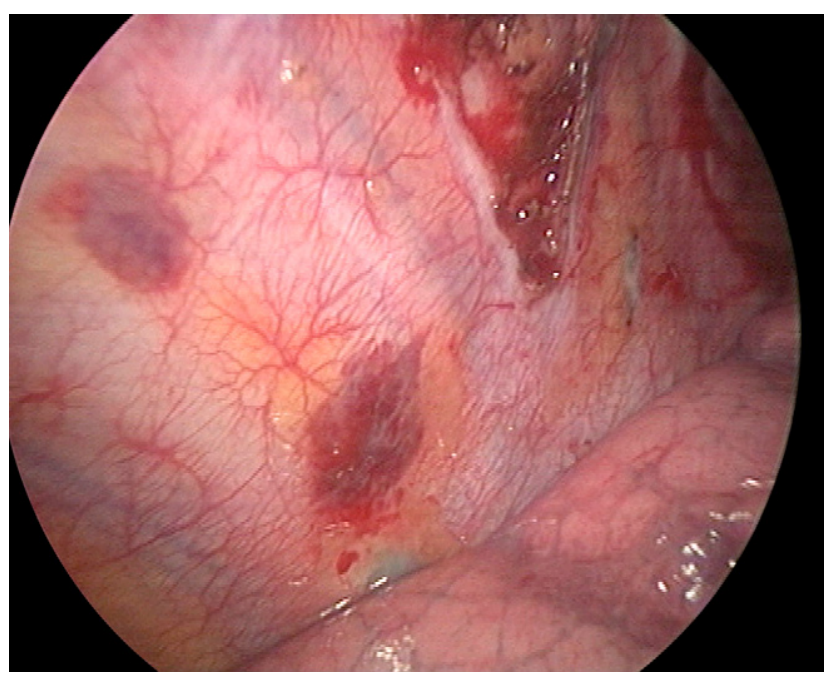

FIGURE 1. Endometrial foci on the parietal pleura, coagulated by argon plasma and removed.

When she presented to the Thoracic Surgery Unit, Ospedale San Paolo, a chest radiograph revealed a complete right pneumothorax. A chest tube was inserted, and the lung was fully reexpanded. However, air leaks persisted for 7 days, and she underwent surgery. During the operation, a 3-port thoracoscopy was performed. There were no pleural adhesions. On the dorsal parietal pleura (Figure 1) at the level of the scapula and on the tendinous center of the diaphragm (Figure 2), some tiny blue brown nodules were detected. They were suspected to be endometriosis, as confirmed by frozen biopsy sections. The diaphragm was accurately inspected, and no holes were found to be present (Figure 2). Moreover, when pulling the diaphragm by forceps, no air was found to be passing through it and the intra-abdominal vacuum persisted. At the apex of the inferior lobe, some blebs were found that appeared to have ruptured. They were removed by stapler. No endometriosis was identified on the parenchyma specimen during histologic study. Pleural and diaphragmatic endometrial foci were coagulated by argon plasma and removed. Pleural abrasion was performed in association with partial pleurectomy corresponding with the internal surface from the first to sixth rib. The clinical course was uneventful, and the patient was discharged on the sixth postoperative day.

After surgery, the mild back pain related to menses disappeared. The patient underwent a new ovarian stimulation 


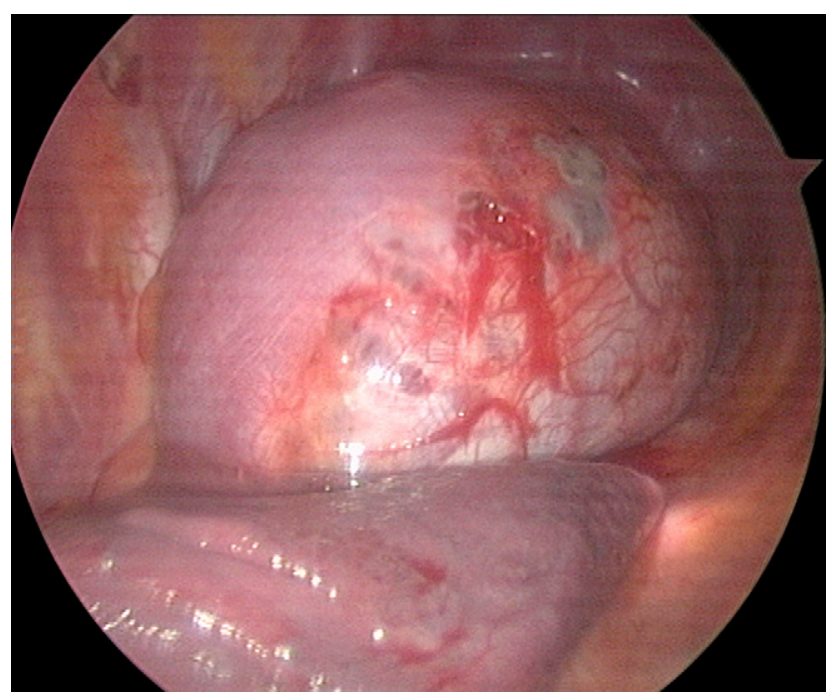

FIGURE 2. Endometrial foci on the diaphragmatic surface. No holes were observed. Pulling the diaphragm by forceps demonstrated that no air was passing through it and that the intra-abdominal vacuum persisted.

with successful IVF-ET cycle 18 months later. No recurrence of pneumothorax occurred at the 22-month follow-up.

\section{DISCUSSION}

There are 3 different theories explaining thoracic endometriosis. The first suggests a retrograde menstruation and secondary implantation on the diaphragmatic surface. ${ }^{1,2}$ The second suggests a coelomic metaplasia of endometrial tissue. ${ }^{5}$ The third is based on metastatic spread of endometrial tissue in the pulmonary parenchyma through the blood vessels. ${ }^{1}$ In our patient, endometrial foci were located on the diaphragmatic surface and parietal pleura but not in the parenchyma specimen. This fact supports the implantation theory.

The relation between endometriosis and pneumothorax is also unclear. Several theories have been proposed, including
(1) the transfallopian ascent air passing thorough diaphragmatic holes, (2) the alveolar rupture caused by prostaglandin-induced bronchiolar constriction, and (3) the perforation of visceral pleura caused by subpleural endometrial tissue proliferating during menses. ${ }^{1}$

In our patient, no fenestration was found in the diaphragm; therefore, no air passage could explain the pneumothorax. This was confirmed by the persistence of air leaks after drainage and the absence of relapse at the 22-month follow-up despite no diaphragmatic surgical repair. We believe that the pneumothorax was caused by the rupture of blebs, probably caused by controlled ovarian hyperstimulation in preparation for the IVF-ET cycle.

\section{CONCLUSIONS}

The surgical management consisting of blebs resection, endometrial foci coagulation, and mechanical pleural abrasion was appropriate to prevent relapse. The endometriosis under the parietal pleura, which was responsible for the thoracic pain during menses, resolved after surgery. Our experience suggests that there are different kinds of catamenial pneumothorax and that, in some cases, bleb resection and pleural abrasion are enough to prevent relapses.

\section{References}

1. Alifano M, Roth T, Broet SC, Schussler O, Magdeleinat P, Regnard JF. Catamenial pneumothorax a prospective study. Chest. 2003;124:1004-8.

2. Marshall MB, Ahmed Z, Kucharczuck JC, Kaiser LR, Shrager JB. Catamenial pneumothorax: optimal hormonal and surgical management. Eur J Cardiothoracic Surg. 2005;27:662-6.

3. Korom S, Canyurt H, Missback A, Schneiter D, Kurrer MO, Haller U, et al. Catamenial pneumothorax revisited: clinical approach and systematic review of the literature. J Thorac Cardiovasc Surg. 2004;128:502-8.

4. Min JK, Claman P, Hughes E. Guidelines for the number of embryos to transfer following in vitro fertilization. Int J Gynaecol Obstet. 2008;102:203-16.

5. Suginami H. A reappraisal of the coelomic metaplasia theory by reviewing endometriosis occurring in unusual sites and instances. Am J Obstet Gynecol. 1991; 165:214-8. 